\title{
Motifs de consultation des patients dans le Service d'Odontostomatologie de l'Hopital de Kayes en 2018, Mali
}

\section{Patients' reasons for consulting dentists in the Odontostomatology Department of Kayes Hospital, Mali}

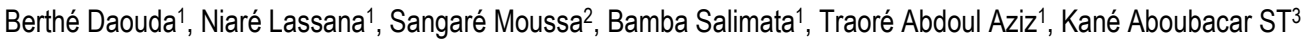

1- Service d'Odontostomatologie de l'Hôpital Fousseyni DAOU de Kayes

2- ICER-MALI/FMPOS, University of Bamako, point G, Bamako,

3- Service d'Odontologie, Hôpital Militaire de Bamako IHB (MALI)

Correspondent: Dr. Daouda BERTHE, Chirurgien-dentiste

Chef Service d'Odontostomatologie de l'Hôpital Fousseyni DAOU de Kayes, Tel : 0022377910885/0022365702728, Email berthed0@gmail.com

\section{RESUME}

Introduction : La cavité buccale peut être le siège de nombreuses pathologies bucco-dentaires responsables de douleurs, de gêne fonctionnels et esthétiques pouvant motiver une consultation dans un service d'odontostomatologie. $\mathrm{Au}$ Mali, les données épidémiologiques sur la santé bucco-dentaire de la population de Kayes sont rares et les données sur les motifs de consultation dans les structures cliniques de prise en charge sont presque inexistantes. L'objectif était de déterminer les différents motifs de consultation pour lesquels les patients sollicitent le service d'odontostomatologie et le type de couverture maladie de l'hôpital de Kayes. Méthodologie : II s'agissait d'une étude transversale descriptive menée auprès des patients ayant consulté le service d'odontostomatologie de l'hôpital de Kayes entre le 1er janvier 2018 et le 31 décembre 2018. Les données ont été extraites du logiciel hôpital management system de l'hôpital de Kayes (HMS) ; qui a servi de base pour l'enregistrement des données. Résultats : Parmi les 2526 patients ayant sollicité le service, 1938(76,72\%) ont été inclus dans l'étude. Le sexe féminin était plus représenté avec 1094 (56,45\%). La tranche d'âge de 15 à 44 ans était la plus représentée 1003 (66,16). Les principaux motifs de consultation étaient : la douleur $1516(78,22 \%)$, la prothèse les prothèses dentaires $106(5,47 \%)$, la mobilité dentaire $67(3,46 \%)$, les soins dentaires conservateurs $56(2,89 \%)$, les traumatismes bucco-dentaires $54(2,80 \%)$, les pathologies infectieuses $48(2,48 \%)$, les consultations de routine $28(1,45 \%)$, le saignement buccal $25(1,29 \%)$, les lésions buccales $15(0,77 \%)$, I'halitose $8(0,4 \%)$ et autres (dyschromie, sécheresse buccale, malposition dentaire, corps étranger inter dentaire, esthétique) 13(0,67\%). Conclusion : Nos données suggèrent que la douleur constitue le principal motif de consultation au service d'odontostomatologie de l'hôpital de Kayes.

Mots clés : Motifs, douleur, parodontopathies, hôpital régional, Kayes Mali.

\section{ABSTRACT}

Introduction: The oral cavity can be the placeof many oral pathologies responsible for pain, functional and aesthetic discomfort that can motivate a consultation in an odontostomatology department. In Mali, epidemiological data on the oral health of the population of Kayes are scarce and the data on the reasons for consultation in the clinical structures of care are almost non-existent. The objective was to determine the different reasons for consultation for which patients request the odontostomatology service at the Kayes hospital. Methods: This was a cross-sectional study of patients years who visited the Odontostomatology Department of Kayes Hospital between January 1, 2018 and December 31,2018 . The data were extracted from the hospital management systemsoftware of Kayes hospital (HMS); which served as a basis for recording data. Results: Among the 2526 patients recorded, 1938 (76.72\%) were included in the study. Female was more represented with $1094(56.45 \%)$. The age group 15-44 was the most represented $1003(66,16 \%)$. The main reasons for consultation were pain $1516(78.22 \%)$, prosthesis 106 $(5.47 \%)$, dental mobility $67(3.46 \%)$, conservative care 56 $(2.89 \%)$, trauma $54(2.80 \%)$, infectious diseases 48 $(2.48 \%)$, routine consultations $28(1.45 \%)$, oral bleeding $25(1.29 \%)$, oral lesions $15(0.77 \%)$, halitosis $8(0.4 \%)$ and others (dyschromia, dry mouth, dental malposition, inter dental foreign body, aesthetic) $13(0.67 \%)$. Conclusion: Our data suggest that pain is the main reason for consultation in the Odontostomatology Department of Kayes Hospital.

Key words: Reasons, pain, caries, periodontal disease, regional hospital, Kayes.

\section{INTRODUCTION}

La cavité buccale est le siège de nombreuses pathologies bucco-dentaires responsables de douleurs, de gêne fonctionnels et esthétiques pouvant motiver une consultation dans un service d'odontostomatologie. A Kayes, première région administrative du Mali et chef-lieu de la région, la prise en charge des affections buccodentaires dont souffre la population s'organise au sein de l'hôpital régional public, le cabinet dentaire de l'armé et le cabinet dentaire privé.

Dans l'étude de BALDE.S à Nancy en 2007(1), la douleur a été le principal motif de consultation soit $(41 \%)$ suivi de l'infection (25\%) ; environ (6\%) pour les traumatismes et $(5 \%)$ pour les mobilités dentaires, les lésions muqueuses ont représenté $(3 \%)$, les problèmes de prothèses (7\%).Une étude réalisée par Ahossi V.et al. 2002 à Dijon en France (2) a montré que $46 \%$ des motifs de consultation sont les douleurs, 33\% représentent les 
pathologies infectieuses, $15 \%$ les traumatismes, $2 \%$ les hémorragies et $4 \%$ des motifs classés autres. Une enquête multicentrique menée par Ahossi V, 2003(3) sur quatre centres hospitaliers en France donne sensiblement les mêmes résultats $45 \%$ des motifs de consultation sont les douleurs, $24 \%$ représentent les pathologies infectieuses, $24 \%$ les traumatismes, $3 \%$ les hémorragies et $4 \%$ des motifs classés autres.

Une étude menée par Guirassy et al au Sénégal (4) rapporte une prévalence de $69,5 \%$ de parodontite contre $23 \%$ de gingivites induites par la plaque. La valeur moyenne des besoins en soins parodontaux des militaires de leur étude était de 2,10 avec un écart type de 0,63. Le détartrage était nécessaire pour $65,68 \%$ des militaires alors que $1,61 \%$ avait besoin de traitements complexes. Ces besoins en soins parodontaux étaient différents selon la catégorie de rang militaire.

Une étude réalisée en 2016 dans le service odontostomatologie de l'hôpital de Kayes Berthé D et al au Mali (5) a relevé les pathologies qui avaient incité les patients à consulter : La carie et/ou ses complications $(50,12 \%)$ étaient au premier plan, affections parodontales $(37,17 \%)$, traumatismes dentaires $(3,96 \%)$, stomatites $(2,74 \%)$, tumeurs $(0,41 \%)$, autres pathologies $(5,60 \%)$.

Peu d'études ont été faites sur l'état bucco-dentaire de la population de Kayes. Parmi les études réalisées aucune ne s'est focalisée sur les motifs de consultation. De ce fait, il n'existe pas peu de données sur les motifs de consultations en odontostomatologie à l'hôpital régional de Kayes. L'objectif de cette étude était d'identifier les différentes raisons qui ont conduit les patients dans le service odontostomatologie de l'hôpital de Kayes.

\section{METHODOLOGIE}

Cette étude transversale descriptive a été réalisée dans le service d'odontostomatologie de l'hôpital de Kayes. L'échantillon était de type non probabiliste. Tous les sujets dont le dossier a été correctement enregistré entre le 1er janvier et le 31 décembre 2018 ont été inclus. Les patients dont les données médico-administratives étaient incomplètes et les anciens patients n'ont pas été inclus dans cette étude. Les données médico-administratives qui étaient complètes et répondaient aux critères suscités ont été inclus dans l'étude.

Le patient était libre de répondre au questionnaire ou par l'intermédiaire de la personne accompagnante, et de se soumettre à l'examen clinique administré par un membre de l'équipe d'étude.

Après l'obtention du consentement verbal auprès du patient ou de ses parents, chaque patient a été consulté et le HMS a servi de base pour recueillir les données. Les données ont été extraites du logiciel HMS sur une fiche d'enquête élaborée à cet effet. Le logiciel Excel a servis de Gestion et d'analyse des données.

\section{RESULTATS}

Durant la période de recueil des données, 2526 patients ont été consultés au service d'odonto-stomatologie de l'hôpital de Kayes pour des problèmes bucco-dentaires. Sur 2526 patients, 1938 patients, dont les données médico-administratives étaient complètes et répondaient aux critères suscités, ont été inclus dans l'étude soit $76,72 \%$ (1938/2526). Le sexe féminin était le plus représenté avec 56,45\% (1094/1938) avec un sex ratio de 1,30 .

Les patients venant de la commune urbaine de Kayes étaient les plus représentés avec $(77,04 \%)$ des cas (Tableau I).

Les patients sans couverture maladie étaient les plus représentés soit $(78,79 \%)$ (Tableau II).

La douleur était le principal motif de consultation soit $78,22 \%$ des cas suivi de prothèse et les réparations de prothèses soit $5,47 \%$ des cas (Tableau III).

La tranche d'âge 15-44 ans était la plus représentée soit $63,42 \%$ suivie de la tranche d'âge $45-59$ ans avec $16,20 \%$ des cas (Tableau IV).

Le sexe féminin représentait $59,62 \%$ des cas pour la douleur comme motif de consultation (Tableau V).

\section{DISCUSSION}

Cette étude réalisée dans le service d'odontostomatologie de l'hôpital Fousseyni Daou de Kayes, a permis d'identifier les différents motifs de consultations. Elle a été conduite auprès de 1938 patients soit $(76,72 \%)$. Nous avons noté une prédominance féminine avec un effectif de 1094 soit $(56,45 \%)$; cette prédominance féminine pourrait s'expliquer par des raisons culturelles et les habitudes alimentaires. Le même constat de prédominance féminine avait été fait dans l'étude de Baldé et al en 2007à Nancy (1) soit (58\%) ; par contre Belhadji Z etalen 2018 (6) ont trouvé une prédominance masculine dans leur étude en Algérie.

La Tranche d'âge de 15 à 44 ans était la plus représentée, soit $63,42 \%$; cette prédominance pourrait s'expliquer par le fait que c'est la tranche d'âge la plus nombreuse en terme de population dans la région et le plus souvent la plus active. Cette tranche d'âge était proche de celle de Belhadji Z et al en 2018 (6) qui était entre 20 et 29 ans soit $(29,4 \%)$.Cette différence pourrait s'expliquer par le fait que les tranches âge adoptées sont différentes.

Dans notre étude les patients résidaient majoritairement dans la commune d'implantation de l'hôpital soit 77,04\%. Cela pourrait s'expliquer par la proximité avec l'hôpital. Les résultats sont concordants avec une autre étude réalisée par Belhadji Z et al en 2018 en Algérie avec 39,6\% (6)

Les patients sans couverture maladie étaient les plus représentés soit $78,79 \%$ et n'étaient que de $10 \%$ dans l'étude de Mickael et al en 2015en France (7). Cette différence pourrait s'expliquer entre autres par la méconnaissance de la couverture maladie, le taux de chômage élevé et le taux élevé de l'activité informelle.

Sur le plan clinique, le motif de consultation le plus fréquent dans cette étude était la douleur $(78,22 \%)$.Cela pourrait s'expliquer par l'inconfort, les insomnies provoquées par les douleurs. 
Ce chiffre est comparable à celui de Belhadji $Z$ et al en2018 en Algérie (6) ; Mickael et al en France (7) qui était de $71 \%$ en 2015 ; de Chiche 1 et al en France (8) en 2007 et en 2010 par Cassam I en France (9) chez les patients des urgences. Cette douleur peut être associée à des pathologies infectieuses, inflammatoires, traumatiques.

Les seconds motifs de consultation de cette étude sont les prothèses dentaires $(5,47 \%)$; cela pourrait s'expliquer par le besoin esthétique et fonctionnel dû à la perte dentaire. Ce chiffre est comparable à celui de Gunepin $M$ et al. en 2014 à Gao au Mali (10) (5,2\%). Les motifs de soins et de perte d'obturation ont représenté $(2,89 \%)$ inférieur à celui de Gunepin et al. (10) en 2014 pays qui étaient de (4,9\%). Ce chiffre pourrait s'expliquer par la méconnaissance des soins dentaire et le manque de professionnels dentaires dans les régions.

Les traumatismes bucco-dentaires ont représenté $(2,80 \%)$, cela pourrait s'expliquer par la mauvaise qualité des routes, l'excès de vitesse et les violences corporelles. Ce chiffre est comparable à celui de KM Bouassalo (11) en 2014 au Mali qui était de (2,3\%), et très inférieur à celui de l'étude réalisée par Ahossi et al (2) en 2002 à Dijon en France ou les traumatismes représentaient (24\%).

Dans notre étude, seulement $(2,48 \%)$ ont évoqué les tuméfactions liées à l'infection comme motif de leur consultation cela était très diffèrent sur le plan clinique, cela pourrait s'expliquer par le fait que la plupart des patients pensent que les tuméfactions buccale et faciale ne sont pas d'origine dentaire mais dû à la sorcellerie. Ce chiffre est inférieur par rapport à l'étude réalisée par Mickael et al. à Paris en France (7) qui était de (11\%) et de celui Gunepin M et al en 2014 à Gao au Mali $(10,3 \%)$ (10).

\section{CONCLUSION}

Cette étude s'inscrit dans le suivi de l'analyse de la patientèle de la consultation au service d'odontostomatologie de l'hôpital de Kayes. Elle a permis de décrire les motifs de consultation au service de d'odontostomatologie de l'hôpital de Kayes. Cette étude a permis d'apporter une estimation des caractéristiques sociodémographiques des patients, de leurs motifs de consultation. Le principal motif de consultation était la douleur.

\section{REFECENCES}

1- BALDE.S- Les urgences et la douleur en odontologie : évaluation statistique de la prise en charge dans un service hôpitalier d'odontologie. Thèse : chir.-Dent. : Nancy : 2007, p.108-109,

2- Ahossi V, DevoizeL, Tazi M, Perrin D. Urgences odontologiques au centre hopitalier universitaire de dijon. Analyse de 12 mois d'activité.

3- Clinic(paris) $2002 ; 23: 165-75$.

4- Ahossi V, Delestan C, Gérard E, Lozza J, Michlowsky S, Moizan $\mathrm{H}$, et al. Urgences odontologiques à l'hôpital. Une inflation de la demande de soins. Lettre Ordre Natl chir Dent 2003; $10.5: 15-7$

5- Guirassy MI, Kane Ast, Thiam D, Diallo Am, Fall N, Diouf A, Seck-Diallo A, Benoist Hm, Évaluation Des Besoins En Soins Parodontaux De Militaires Sénégalais Sélectionnés Pour Une Mission De Maintien De La Paix Au Mali, Rev Col OdontoStomatol Afr Chir Maxillo-Fac, 2020, 27, 1, 27-32.

6- Berthé D. Rapport de stage probatoire hôpital Fousseyni Daou odontostomatologie 2015-2016; 22 $p$ : Archive secrétariat général Hôpital Fousseyni Daou

7- Belhadji Z, Belkacem B, Bensafi S, les urgences en odontostomatologie au niveau du CHU Tlemcen : caractéristiques épidemiologiques, cliniques et radiologiques. Mémoire : chir.-dent. : Tlemcen : université Abou Bekr Belkaîd ; 2017-2018.

8- Mickael A, Claude Bernard W: Caractéristiques des patients consultant aux urgences odontologiques, motifs de recours et prise en charge, Enquête réalisée dans l'unité d'urgence du Groupe Hospitalier Pitié Salpétrière. consultable hpps://www.aonews-lemag.fr consulté le 29 mars 2019:

9- Chiche L, Motifs de consultation et prise en charge des patients accueillis en urgence ; enquête dans l'unité fonctionnelle d'urgence du service d'odontologie du groupe hospitalier Pitiésalpêtrière.2007,th :chir Dent : université Paris 72007. consultable hpps://www.aonews-lemag.fr consulté le 29 mars 2019

10- Cassam I, Evaluation de la satisfaction des patients : enquête dans l'unité fonctionnelle d'urgence du service d'odontologie du groupe hospitalier Pitiésalpêtrière.2010,th :chir Dent : université Paris 72010. consultable hpps://www.aonews-lemag.fr consulté le 29 mars 2019

11- Gunepin M, Benmansour A, Derache F, Maresca S, Blatteau J-E, Risso J.-J. motifs de consultation en urgence au cabinet dentaire du groupement médicochirurgical de Gao : quelles leçons en tirer ? Rg journal médecine et armées ,2015,43,4,345-351 https://www.researchgate.net/publication/28332172 9_Motifs_de_consultation_en_urgence_au_cabinet dentaire du groupement medicochirurgical de Gao quelles lecons en tirer Consulté le 03 avril 2019

12- K.M.Bouassalo, S.Adam,H.D. Sama, B.E. Kebina,H. Bissa et M. Gunepin.Togo :Problèmes dentaires rencontrés par les forces Togolaises déployées au Mali. $\mathrm{Rg}$ Journal médecine et armées, $2017,45,3,343-348$.

\section{Liste des tableaux}

Tableau I : Répartition des patients selon le lieu de résidence 
MALI SANTE PUBLIQUE 2020 SOMASAP

\begin{tabular}{lcc}
\hline Lieu de résidence & $\mathbf{n}$ & $\%$ \\
\hline Commune urbaine de Kayes & 1493 & 77,04 \\
Cercle de Kayes & 271 & 13,97 \\
Région de Kayes & 146 & 7,53 \\
\hline
\end{tabular}

\begin{tabular}{lcc}
\hline Lieu de résidence & $\mathbf{n}$ & $\%$ \\
\hline $\begin{array}{l}\text { Hors région de Kayes et autres } \\
\text { payes }\end{array}$ & 28 & 1,46 \\
Total & 1938 & 100 \\
\hline
\end{tabular}

Tableau II : Répartition des patients selon le type de couverture maladie

\begin{tabular}{lcccccccc}
\hline $\begin{array}{c}\text { Type de } \\
\text { couverture } \\
\text { maladie }\end{array}$ & $\begin{array}{c}\text { Sans } \\
\text { couverture } \\
\text { maladie }\end{array}$ & AMO & RAMED & MUTUEL & $\begin{array}{c}\text { Autres } \\
\text { assurances }\end{array}$ & $\begin{array}{c}\text { Demi- } \\
\text { tarif }\end{array}$ & Gratuité & Total \\
\hline Nombre & 1527 & 203 & 9 & 22 & 11 & 10 & 156 & 1938 \\
Pourcentage & 78,79 & 10,47 & 0,46 & 1,14 & 0,57 & 0,52 & 8,05 & 100 \\
\hline
\end{tabular}

Tableau IV : Répartitions des patients selon le motif de consultation

\begin{tabular}{lcc}
\hline Motif de Consultation & $\mathbf{n}$ & $\%$ \\
\hline Douleurs & 1516 & 78,22 \\
Prothèse et réparation prothèse & 106 & 5,47 \\
Mobilité dentaire & 67 & 3,46 \\
Soins dentaire & 56 & 2,89 \\
Traumatismes & 54 & 2,80 \\
Pathologie infectieuse & 48 & 2,48 \\
Consultation de routine & 28 & 1,45 \\
Saignement & 25 & 1,29 \\
Lésion buccale & 15 & 0,77 \\
Autre motif de consultation & 13 & 0,67 \\
Halitose & 8 & 0,4 \\
Blocage ATM & 2 & 0,1 \\
Total & 1938 & 100 \\
\hline
\end{tabular}

${ }^{*}$ Consultation de routine : Bilan dentaire et soins préventifs

* Lésion buccale : Ulcères buccale d'origine traumatique, infectieuse, systémique, dermatologique et aphteuse

Tableau V : Répartition des patients selon les motifs de consultation par tranche d'âge

\begin{tabular}{lcccccc}
\hline Age (année) & $1-4$ & $\mathbf{5 - 1 4}$ & $\mathbf{1 5 - 4 4}$ & $\mathbf{4 5 - 5 9}$ & $\mathbf{6 0}$ & Total \\
Motif de consultation & & & & & & \\
\hline Douleur & 4 & 130 & 1003 & 230 & 149 & 1516 \\
& $(0,26)$ & $(8,58)$ & $(66,16)$ & $(15,17)$ & $(9,83)$ & $100 \%$ \\
Traumatisme & 1 & 13 & 32 & 1 & 7 & 54 \\
& $(1,85)$ & $(24,07)$ & $(59,26)$ & $(1,85)$ & $(12,96)$ & $100 \%$ \\
Pathologie infectieuse & 1 & 7 & 27 & 10 & 3 & 48 \\
& $(2,08)$ & $(14,58)$ & $(56,25)$ & $(20,83)$ & $(6,25)$ & $100 \%$ \\
Mobilité dentaire & 0 & 0 & 21 & 23 & 23 & 67 \\
& $(0)$ & $(0)$ & $(31,34)$ & $(34,33)$ & $(34,33)$ & $100 \%$ \\
Prothèse dentaires & 0 & 0 & 48 & 25 & 33 & 106 \\
& $(0)$ & $(0)$ & $(45,28)$ & $(23,58)$ & $(31,13)$ & $100 \%$ \\
Soins conservateurs & 0 & 5 & 42 & 9 & 0 & 56 \\
& $(0)$ & $(8,93)$ & $(75)$ & $(16,07)$ & $(0)$ & $100 \%$ \\
Saignement & 1 & 0 & 18 & 4 & 2 & 25 \\
& $(4)$ & $(0)$ & $(72)$ & $(16)$ & $(8)$ & $100 \%$ \\
Consultation de routine & 0 & 1 & 19 & 6 & 2 & 28 \\
& $(0)$ & $(3,57)$ & $(67,85)$ & $(21,43)$ & $(7,14)$ & $100 \%$ \\
Lésion buccale & 3 & 1 & 5 & 2 & 4 & 15 \\
& $(20)$ & $(6,67)$ & $(33,33)$ & $(13,33)$ & $(26,67)$ & $100 \%$ \\
Halitose & 0 & 0 & 7 & 1 & 0 & 8 \\
\hline
\end{tabular}




\section{MALI SANTE PUBLIQUE 2020 SOMASAP}

\begin{tabular}{lcccccc}
\hline Age (année) & $\mathbf{1 - 4}$ & $\mathbf{5 - 1 4}$ & $\mathbf{1 5 - 4 4}$ & $\mathbf{4 5 - 5 9}$ & $\mathbf{6 0}$ & Total \\
Motif de consultation & & & & & & \\
\hline \multirow{3}{*}{ Blocage ATM } & $(0)$ & $(0)$ & $(87,5)$ & $(12,5)$ & $(0)$ & $100 \%$ \\
& 0 & 0 & 0 & 0 & 2 & 2 \\
Autres motif & $(0)$ & $(0)$ & $(0)$ & $(0)$ & $(100)$ & $100 \%$ \\
\multirow{2}{*}{ Total } & 0 & 0 & 7 & 3 & 3 & 13 \\
& $(0)$ & $(0)$ & $(53,84)$ & $(23,08)$ & $(23,08)$ & $100 \%$ \\
& 10 & 157 & 1229 & 314 & 228 & 1938 \\
\hline
\end{tabular}

Tableau V : Répartition des motifs de consultations selon le sexe

\begin{tabular}{lccccc}
\hline \multirow{2}{*}{ Motif de consultation } & \multicolumn{2}{c}{ Femme } & \multicolumn{2}{c}{ Homme } & \multirow{2}{*}{ Total } \\
\cline { 2 - 5 } & $\mathbf{n}$ & $\%$ & $\mathbf{n}$ & $\%$ & \\
\hline Douleurs & 902 & 59,62 & 614 & 40,58 & 1513 \\
Traumatismes & 11 & 20,37 & 43 & 79,63 & 54 \\
Pathologie infectieuse & 27 & 56,25 & 21 & 43,75 & 48 \\
Mobilité dentaire & 31 & 46,27 & 36 & 53,73 & 67 \\
Prothèse et Réparation de Prothèse & 51 & 48,11 & 55 & 51,89 & 106 \\
Soins & 26 & 46,43 & 30 & 53,57 & 56 \\
Saignement & 8 & 32 & 17 & 68 & 25 \\
Consultation de routine & 15 & 53,57 & 13 & 46,43 & 28 \\
Lésion buccale & 6 & 40 & 9 & 60 & 15 \\
Halitose & 7 & 87,5 & 1 & 12,5 & 8 \\
Blocage ATM & 2 & 100 & 0 & 0 & 2 \\
Autres motif de consultation & 8 & 61,54 & 5 & 38,46 & 13 \\
Total & 1094 & $\mathbf{5 6 , 4 5}$ & $\mathbf{8 4 4}$ & $\mathbf{4 3 , 5 5}$ & $\mathbf{1 9 3 8}$ \\
\hline
\end{tabular}

